\title{
Prophylactic cranial irradiation in SCLC
}

\author{
Rafal Suwinski \\ Radiotherapy and Chemotherapy Clinic and Teaching Hospital, Maria Sklodowska-Curie National Research Institute of Oncology, Gliwice Branch, \\ Poland \\ Correspondence to: Rafal Suwinski, MD, PhD. Radiotherapy and Chemotherapy Clinic and Teaching Hospital, Maria Sklodowska-Curie National \\ Research Institute of Oncology, Wybrzeze Armii Krajowej 14, 44-100 Gliwice, Poland. Email: rafal.suwinski@io.gliwice.pl.
}

\begin{abstract}
Prophylactic cranial irradiation (PCI) has well established place in therapy for patients with limited-disease small cell lung cancer who responded to treatment. The data from randomized trials document that PCI reduces brain metastases rate from approximately $60 \%$ to $30 \%$, and increases 3 -year overall survival by approximately 5\%. Currently, the dose of 25 Gy in 10 fractions is considered as standard. In attempt to reduce neuropsychological sequelae attributable to PCI hippocampal sparing techniques are employed. The existing studies suggest the benefit of hippocampal sparing in limiting memory and higher neurocognitive function losses, but with a risk of failures in the spared region. Ongoing studies will further validate the role of hippocampal sparing, both in terms of toxicity reduction and metastases prevention. PCI for patients who have undergone resection for stage I small cell lung cancer (SCLC) is not recommended, PCI may be, however, associated with a favourable outcome in SCLC patients who have undergone complete surgery in stages II-III. The role of PCI in extensive-disease (ED) SCLC has been evolving. Most recent evidence indicate that $\mathrm{PCI}$ is controversial in ED patients with response to initial chemotherapy and absence of brain metastases confirmed by contrast-enhanced MRI. The patients who do not receive PCI, must, however, receive periodic MRI examination during follow-up, i.e., remain under active surveillance with access to radiotherapy at brain relapse. The assessment of safety and effectiveness of hippocampalsparing PCI, with or without drug neuroprotection in consideration of diverse combinations of radiotherapy, chemotherapy and immunotherapy create a background for future directions of research.
\end{abstract}

Keywords: Small cell lung cancer (SCLC); prophylactic cranial irradiation; hippocampal sparing; review

Submitted Mar 07, 2020. Accepted for publication Aug 11, 2020.

doi: $10.21037 /$ tlcr-2020-rtm-05

View this article at: http://dx.doi.org/10.21037/tlcr-2020-rtm-05

\section{Rationale for prophylactic cranial irradiation in small cell lung cancer (SCLC)}

The concept of prophylactic cranial irradiation for patients with small cell lung cancer has been developed as a consequence of progress in combined modality treatment for thoracic disease: in spite of high responses to initial therapy in the thorax approximately $60 \%$ of the patients developed metastases in the brain (1). It was commonly assumed that blood-brain barrier was responsible for inability of chemotherapy to eliminate subclinical cancer in the brain. While the concept of blood-brain barrier has been recently modified by demonstrating the presence of functional lymphatic vessels in the dural sinuses (2), clearly, endothelial, epithelial and glial brain barriers establish compartments that differ with regard to their accessibility to the drugs. This established the biological rationales for prophylactic cranial irradiation: the direct cytotoxic effect of radiotherapy on subclinical brain metastases may be further enhanced by changes in blood-brain barrier permeability induced by radiotherapy (3).

\section{PCI in limited-stage SCLC}

\section{Early trials on PCI in SCLC}

The early trials on PCI in SCLC focused, primarily, on patients in complete remission after treatment for thoracic 
disease. Several trials addressed this issue: while all of them demonstrated significant reduction of metastases in the brain the impact of PCI on overall survival had not been demonstrated. In 1999 Aupérin et al. (4) performed metaanalysis of individual data on 987 patients recruited in seven randomized trials on patients with SCLC in complete remission. Out of 987 patients included $86 \%$ had limited disease and $14 \%$ had extensive disease, based on staging procedures that are nowadays considered outdated. Notably, the evaluation of complete response was based on chest radiographs and not CT scans in most trials. PCI reduced the cumulative incidence of brain metastasis at three years, from $58.6 \%$ in the control group to $33.3 \%$ in the treatment group, which corresponded to a pooled relative risk of 0.46 . Most importantly, the meta-analysis demonstrated an absolute increase in survival of $5.4 \%$ at three years, from $15.3 \%$ in the control group to $20.7 \%$ in the treatment group. The survival benefit from PCI was statistically significant, with a pooled relative risk of 0.84 . Based on the outcome of this meta-analysis PCI was introduced as part of the standard treatment of patients with SCLC in complete remission.

\section{PCI for early $L S-S C L C$}

The existing evidence support use of surgery in stage I SCLC (5). Occasionally, patients with more advanced disease also have surgery, although its routine use is not supported by the existing data. Postoperative chemotherapy is, generally, recommended in patients who underwent surgery for SCLC (6). For patients with stage I or II node negative LS-SCLC who are medically inoperable, either SBRT or conventional fractionation is recommended and chemotherapy should be delivered before or after SBRT (7).

As for PCI its benefit is unknown for patients who have undergone resection, SBRT or conventionally fractionated radiotherapy for early stage SCLC (7-9). The meta-analysis of five retrospective studies revealed, however, that PCI might be associated with a favourable survival advantage and reduced BM risk in SCLC patients who underwent complete surgery in stages II-III (8).

Some differences may be noticed between the recent guidelines of ESTRO, ASTRO and NCCN regarding use of PCI in early LS SCLC.

ESTRO experts recommend prophylactic cranial irradiation and thoracic radiotherapy for patients with resected SCLC and positive lymph nodes (10). For elderly patients with resected node negative SCLC, most of the
ESTRO experts do not recommend thoracic radiotherapy or prophylactic cranial irradiation.

ASTRO guidelines conditionally not recommend PCI for patients with stage I SCLC (7). Instead of PCI, surveillance using brain MRI with contrast can serve as an alternative.

NCCN Guidelines state that the benefit of PCI is unknown in patients who have undergone complete resection for pathologic stage I-IIA (T1-2N0M0) SCLC (11). PCI or brain surveillance should be considered for N0. These patients have a lower risk of developing metastases than patients with more advanced LS SCLC, and may not benefit from PCI.

\section{Dose-response for PCI}

The dose-response curves derived from the data on therapy for detectable cancer are threshold-sigmoid, indicating that low radiation doses cannot sterilize the overt tumour, that may contain $10^{9}-10^{11}$ cells. By contrast, the doseresponse curve for control of subclinical metastases (1-10 cells) is linear and shallow, and extrapolates to a dose intercept not demonstrably different from zero, indicating that low radiation doses may sterilize small subclinical cancer deposits (12). Such shallow dose-response was also demonstrated in PCI in SCLC. Review of dose-response in historical trials on PCI suggested, however, displacement of the dose threshold if PCI was applied with considerable time delay relative to treatment for primary cancer (13). This, likely, reflect growth of residual micrometastases between treatment for the primary and prophylactic irradiation.

The spectrum of total radiation doses used in the trials that were included in Aupérin et al. meta-analysis allowed to analyse dose-response effect in PCI (4). There was a significant trend toward a lower risk of brain metastasis as the radiation dose increased. The effect of treatment on survival did not, however, differ significantly according to the total dose. Also, there was a significant trend toward a greater reduction in the incidence of brain metastasis in patients randomized sooner after induction therapy than in those randomized later.

While dose-response characteristics of PCI might suggest the benefit from use of high radiation doses and early administration of PCI, such inference must be confronted against the toxicity of treatment. To address this important issue an international consortium of EORTC, RTOG, and IFCT performed a randomised clinical trial 
that compared the effects of standard versus higher PCI doses (14). The trial recruited 720 patients with limitedstage SCLC in complete remission after chemotherapy and thoracic radiotherapy. They were randomized to receive 25 Gy in 10 daily fractions $v s .36$ Gy delivered using either conventional fractionation (18 daily fractions of $2 \mathrm{~Gy}$ ) or accelerated fractionation (24 fractions in 16 days with two daily sessions of $1.5 \mathrm{~Gy})$. There was no significant difference in the 2-year incidence of brain metastases between the standard PCI dose group and the higher-dose group (29\% vs. $23 \%$ ). 2 -year overall survival was $42 \%$ in the standarddose group and $37 \%$ in the higher-dose group. The lower overall survival in the higher-dose group was attributed to increase in mortality. Also, a pooled analysis by the North Central Cancer Group revealed a survival difference between PCI total dose of 25 and $30 \mathrm{~Gy}$, in favour of lower dose, likely associated with higher rates of adverse events in the 30 Gy group (15). These results established 25 Gy in 10 fractions as the standard dose for PCI in SCLC.

\section{Timing of PCI}

As mentioned, the dose-response characteristics for PCI may, potentially, favour its early use, considering more effective reduction of the cumulative incidence of brain metastasis $(4,13)$. The potential benefit from early administration of PCI must be, however, carefully weight against the potential increase in toxicity of such approach. While changes in blood-brain barrier permeability induced by radiotherapy may enhance the effectiveness of chemotherapy in reduction of brain metastases, the same mechanisms may result in detrimental brain injury (3). Also, early use of PCI may, potentially, interfere with haematological tolerance of combined treatment.

A historical study that addressed the timing of PCI suggested no difference in the frequency of brain metastases between PCI performed at the start of induction treatment and PCI delivered 6 weeks later, concurrently with chemotherapy (16). The applicability of these results to the current practice is, however, problematic due to obsolete treatment schedules and techniques used at this time. More recent study demonstrated a significant decrease in intracranial recurrence when PCI was performed during chemoradiotherapy as opposed to following chemoradiotherapy. The observed incidence of brain metastases was $7.3 \%$ in patients who received "early" PCI, and $20 \%$ in patients after "late" PCI (17). Early PCI was defined as delivered during chemoradiotherapy, while PCI performed after chemoradiotherapy was defined as "late" in this study. A subsequent publication revealed, however, that no difference with respect to overall survival was observed when "early" and "late" PCI were compared (18). Both studies $(17,18)$ were retrospective, which limits strength of the conclusions provided. A similar inference was provided by another retrospective study that included 399 patients with limited-stage SCLC (19). "Early" and "late" PCI groups were separated using the median time interval between the start of primary chemotherapy and the start of PCI. No significant difference was identified between the early and late PCI groups, either in the incidence of brain metastases or the overall survival (19).

Considering the existing data on "early" versus "late" use of PCI the protocol of the CONVERT trial, that recently established the optimal dose-fractionation schedule for thoracic radiotherapy in SCLC, recommended to deliver PCI to patients without evidence of progressive disease on the CT scan and with no clinical evidence of brain metastases no later than 6 weeks after the last cycle of chemotherapy (20). In general, however, the data on timing of PCI are limited and mostly retrospective, and the definition of "early" and "late" PCI differs among the studies. It suggests the necessity of new research that would address this important issue. Considering these limitations and in view of the existing publications and guidelines $(7,11)$ the standard of care timing of PCI and CTRT might be proposed as follows:

* In concurrent CTRT PCI is delivered after completion of CTRT: the optimal interval is not, however, defined;

* In sequential CTRT (whenever clinical situation justifies use of sequential treatment) PCI is delivered after chemotherapy and generally at the same time as thoracic RT.

\section{PCI in extensive disease}

In 2007 Slotman et al. published results of phase III trial that evaluated the effectiveness and tolerance of PCI in extensive small-cell lung cancer (21). Only the patients who responded after four to six cycles of initial chemotherapy were included and brain imaging was not mandated. Inclusion criteria allowed to enrol the patients in WHO performance status of $0-2$, with an interval of no more than 5 weeks between the last cycle of chemotherapy and randomization. Total PCI radiation doses were in range of 20-30 Gy. Patients in the PCI group had a lower risk of 
symptomatic brain metastases, compared to control (1-year cumulative risk of brain metastases was $14.6 \%$ vs. $40.4 \%$, HR 0.27). PCI was associated with significant increase in overall survival (1-year survival rate was $27.1 \%$ vs. $13.3 \%$, HR 0.68). As mentioned before, adverse events of PCI were observed in this trial, the global quality of life of the patients was, however, not significantly impaired in treatment group.

An important limitation to the trial by Slotman et al. (21) is that the protocol did not necessitate CT/MRI screening for brain metastases before PCI. Therefore, some individuals with asymptomatic (but radiologically detectable) brain metastases could be, potentially, enrolled.

To address this issue Takahashi et al. (22) performed a randomized trial which recruited the patients with extensive-disease SCLC. The response was assessed after completion of two or more cycles of initial platinum-based doublet chemotherapy, the absence of brain metastases confirmed by gadolinium-enhanced MRI was required for recruitment. Patients in both trial arms, irrespective of the presence or absence of neurological symptoms, were required to have brain MRI at 3-month intervals up to 12 months and at 18 and 24 months after enrolment. Patients who did not receive PCI were, thus, under active surveillance, while the patients in treatment arm received PCI at a total dose of 25 Gy delivered in 10 fractions. The cumulative incidence of brain metastases at 12 months were $32.9 \%$ in the PCI group vs. 59.0\% in "observation" arm, the difference was statistically significant. Progression-free survival did not, however, differ significantly between the two groups. Likewise, no significant difference between groups in overall survival was seen: median overall survival was 11.6 months in the PCI and 13.7 months in the "observation" arm (HR 1.27). Radiotherapy for brain metastases was given in $83 \%$ of the patients with brain metastases in the observation group, and this proportion was higher than in PCI arm. A limitation of this research was lack of QuL/neurotoxicity data It was concluded from this trial that prophylactic cranial irradiation is controversial for patients with extensive-disease small-cell lung cancer with response to initial chemotherapy and a confirmed absence of brain metastases. The patients who do not receive PCI, must, however, receive periodic MRI examination during follow-up, i.e., remain under active surveillance. PCI in ED SCLC should be, thus, replaced by active surveillance with access to salvage conformal/ stereotactic brain radiotherapy. Clearly, this finding has impacted decision making regarding PCI in ED-SCLC (23) and found reflection in clinical guidelines.
The role of PCI in extensive disease has been further challenged in the context of immunotherapy use. The IMpower133 trial reported a survival benefit to atezolizumab for ES-SCLC (24). The protocol of this trial allowed, bud did not require PCI use: eventually only $10 \%$ of the patients received PCI during the maintenance phase of immunotherapy. Likewise, the protocol of a randomized multicenter phase 3 study of nivolumab alone or in combination with ipilimumab in patients with ED SCLC (CheckMate 451, ClinicalTrials.gov Identifier: NCT02538666) defines that PCI may be offered to subjects following the completion of first-line chemotherapy, per local standard of care. Recently presented retrospective data suggest, nevertheless, that PCI use has declined after introduction of immunotherapy for patients with ED SCLC (25). Clearly, new studies are required to assess safety and efficacy of PCI in ED SCLC patients who receive immunotherapy.

\section{Toxicity of PCI}

It has been early recognized that use of PCI may carry a considerable neurotoxicity in long-term survivors of SCLC (26).

On the other hand, a systematic review of the literature on health-related quality of life in SCLC patients revealed, that the diagnosis of SCLC, by itself, negatively affects quality of life, in comparison to the normal population (27). The quality of life appeared to be affected not only by extent of the disease, by also by response to treatment, with better quality of life in good responders.

In a randomized trial of prophylactic cranial irradiation compared with observation in patients with locally advanced non-small-cell lung cancer PCI was delivered to a total dose of $30 \mathrm{~Gy} / 15$ fractions, once daily (28). The neurocognitive and quality-of-life analysis did not find any significant differences in global cognitive function or QOL after PCI, but there was a significant decline in memory (HVLT) at 1 year. One may notice that the PCI dose used in this trial was higher than currently recommended.

Slotman et al. performed a trial on PCI in extensive disease small-cell lung cancer including the analysis of short-term health-related quality of life and patient reported symptoms (29). The PCI doses of 20 Gy in 5 or 8 fractions, 24 Gy in 12 fractions, 25 Gy in 10 fractions, or 30 Gy in 10 or 12 fractions were allowed. There was a negative impact of PCI on selected HRQOL scales with largest difference between the arms for fatigue and hair loss. For global health 
status, the observed mean difference was not significant.

In a randomized study that evaluated optimal radiation dose of PCI a mild deterioration across time of communication, intellectual and memory deficit were generally observed, with no significant difference between 25 and 36 Gy groups (30).

Two other randomized controlled trials have compared PCI to no PCI and reported on neurocognitive function $(31,32)$. The outcome of these trials has been summarized and discussed elsewhere (33). In general, no evidence of major impairment attributable to PCI was demonstrated in these trials.

Several confounding factors can impact on neurocognition after PCI. This include the disease itself, radiation dose fractionation, concurrent or sequential use of chemotherapy, age, effects of tobacco smoking, paraneoplastic syndromes, or presence of undiagnosed micrometastases. Patients may also have underlying symptoms of depression, decreased attention, memory and problem-solving ability which further affect the overall impact of PCI on neurocognition. These factors contribute for patient concerns about neurotoxicity of brain radiotherapy, which appears to be the most common reason for PCI omission in routine therapy for SCLC (34).

Current guidelines do not recommend PCI in patients with neurocognitive impairment or those in poor general performance status. Also, the neurotoxicity of PCI should be of particular concern among elderly patients ( $>60$ years) $(7)$. An active MRI surveillance for brain metastases might be the choice for such individuals.

\section{Attempts to reduce the toxicity of PCI}

Some studies attempted to detect brain structural changes in patients with lung cancer who were undergoing PCI for SCLC (35). In general, hippocampal region was identified as responsible for memory and higher neurocognitive function losses after PCI. Such observation created the rational background for attempts to reduce the toxicity of PCI by sparing this region.

Intensity-modulated radiotherapy allows to avoid irradiation of the hippocampal neural stem-cell compartment during whole brain radiotherapy. Such technique has been investigated both for patients with brain metastases from various primary sites, as for PCI in patients with SCLC. A phase II trial that recruited 113 patients (including 42 available at 4 months) demonstrated, that in patients with brain metastases, conformal avoidance of the hippocampus during WBRT was associated with preservation of memory and QOL as compared with historical series (36). A prospective study of hippocampalsparing PCI in limited-stage SCLC was also performed, and included 20 patients (37). The outcome of this study suggests the benefit of hippocampal sparing in limiting the neuropsychological sequelae of brain radiation, but with a risk of failures in the spared region. Two studies analysed the incidence of hippocampal metastasis $(38,39)$. Guo et al. (38), based on retrospective analysis of the clinical characteristics and patterns of brain metastases detected at the time of diagnosis or during the followup in patients with SCLC, concluded that the incidence of perihippocampal metastases (12\%) may be acceptably low enough to justify hippocampal-sparing techniques for SCLC. A similar conclusion was provided by Kundapur et al. (39), based on evaluation of risk of hippocampal metastases in small cell lung cancer at presentation and after WBRT. Out of 59 patients presenting with de novo brain metastases, 3 patients ( $5 \%$ ) had hippocampal metastases. An interesting case report (40) clearly indicate, however, necessity of further validation of hippocampal-sparing PCI both in terms of neurocognitive protection and brain metastases prevention. A large clinical trial on this subject (ClinicalTrials.gov identifier NCT02635009) started recruitment of the patients. The early outcome of two other studies has been presented at ASTRO 2019 (41,42).

It is important to recognize attempts to improve the tolerance of PCI by drug neuroprotection. These attempts were discussed in recent review on patient selection, efficacy and outcomes of PCI in SCLC (43). An evidence on utility of such approach provide results of RTOG 0614 trial that studied memantine for the prevention of cognitive dysfunction in patients receiving whole-brain radiotherapy (44). Memantine is a drug that was studied in patients with neurodegenerative disorders, including Alzheimer disease. More recent results provide Phase III Trial NRG Oncology CC001 trial on hippocampal avoidance during wholebrain radiotherapy plus memantine for patients with brain metastases (45). It was concluded, based on outcome of this trial, that hippocampal avoidance WBRT plus memantine better preserved cognitive function and patient-reported symptoms than WBRT plus memantine, with no difference in intracranial PFS and OS. Although both of these trials $(44,45)$ refer to radiotherapy for patients with detectable brain metastases, and not to PCI, the recent NCCN guidelines for the management of SCLC recommend considering memantine during and after administration of 
PCI (11).

It appears that the assessment of safety and effectiveness of hippocampal-sparing PCI, with or without drug neuroprotection in consideration of diverse combinations of radiotherapy, chemotherapy and immunotherapy create a robust background for future directions of research in this field.

\section{Acknowledgments}

Funding: None.

\section{Footnote}

Provenance and Peer Review: This article was commissioned by the Guest Editors (Jacek Jassem and Rafal Dziadziuszko) for the series "Radiotherapy in thoracic malignancies" published in Translational Lung Cancer Research. The article was sent for external peer review organized by the Guest Editors and the editorial office.

Conflicts of Interest: The author has completed the ICMJE uniform disclosure form (available at http://dx.doi. org/10.21037/tlcr-2020-rtm-05). The series "Radiotherapy in thoracic malignancies" was commissioned by the editorial office without any funding or sponsorship. The author has no other conflicts of interest to declare.

Ethical Statement: The author is accountable for all aspects of the work in ensuring that questions related to the accuracy or integrity of any part of the work are appropriately investigated and resolved.

Open Access Statement: This is an Open Access article distributed in accordance with the Creative Commons Attribution-NonCommercial-NoDerivs 4.0 International License (CC BY-NC-ND 4.0), which permits the noncommercial replication and distribution of the article with the strict proviso that no changes or edits are made and the original work is properly cited (including links to both the formal publication through the relevant DOI and the license). See: https://creativecommons.org/licenses/by-nc-nd/4.0/.

\section{References}

1. Komaki R, Cox JD, Whitson W. Risk of brain metastasis from small cell carcinoma of the lung related to length of survival and prophylactic irradiation. Cancer Treat Rep
1981;65:811-4.

2. Louveau A, Smirnov I, Keyes TJ, et al. Structural and functional features of central nervous system lymphatic vessels. Nature 2015;523:337-41.

3. van Vulpen M, Kal HB, Taphoorn MJ, et al. Changes in blood-brain barrier permeability induced by radiotherapy: implications for timing of chemotherapy? Oncol Rep 2002;9:683-8.

4. Aupérin A, Arriagada R, Pignon JP, et al. Prophylactic cranial irradiation for patients with small-cell lung cancer in complete remission. Prophylactic Cranial Irradiation Overview Collaborative Group. N Engl J Med 1999;341:476-84.

5. Hoda MA, Klikovits T, Klepetko W. Controversies in oncology: surgery for small cell lung cancer? It's time to rethink the case. ESMO Open 2018;3:e000366.

6. Yang CF, Chan DY, Speicher PJ, et al. Role of adjuvant therapy in a population-based cohort of patients with early-stage small-cell lung cancer. J Clin Oncol 2016;34:1057-64.

7. Simone CB 2nd, Bogart JA, Cabrera AR, et al. Radiation Therapy for Small Cell Lung Cancer: An ASTRO Clinical Practice Guideline. Pract Radiat Oncol 2020;10:158-73.

8. Yang Y, Zhang D, Zhou X. Prophylactic cranial irradiation in resected small cell lung cancer: A systematic review with meta-analysis. J Cancer 2018;9:433-9.

9. Eze C, Roengvoraphoj O, Manapov F. Prophylactic cranial irradiation in resected early stage small cell lung cancer. Int J Radiat Oncol Biol Phys2017;98:612-4.

10. The role of postoperative thoracic radiotherapy and prophylactic cranial irradiation in early stage small cell lung cancer: Patient selection among ESTRO experts. Radiother Oncol 2020;145:45-8.

11. NCCN Clinical Practice Guidelines in Oncology. Small Cell Lung Cancer. Version 1.2021-August 42020.

12. Withers HR, Peters LJ, Taylor JM. Dose-response relationship for radiation therapy of subclinical disease. Int J Radiat Oncol Biol Phys 1995;31:353-9.

13. Suwinski R, Lee SP, Withers HR. Dose-response relationship for prophylactic cranial irradiation in small cell lung cancer. Int J Radiat Oncol Biol Phys 1998;40:797-806.

14. Le Péchoux C, Dunant A, Senan S, et al. Standard-dose versus higher-dose prophylactic cranial irradiation (PCI) in patients with limited-stage small-cell lung cancer in complete remission after chemotherapy and thoracic radiotherapy (PCI 99-01, EORTC 22003-08004, RTOG 0212, and IFCT 99-01): a randomised clinical trial. Lancet 
Oncol 2009;10:467-74.

15. Schild SE, Foster NR, Meyers JP, et al. North Central Cancer Treatment Group. Prophylactic cranial irradiation in small-cell lung cancer: findings from a North Central Cancer Treatment Group Pooled Analysis. Ann Oncol 2012;23:2919-24.

16. Perez CA, Krauss S, Bartolucci AA, et al. Thoracic and elective brain irradiation with concomitant or delayed multiagent chemotherapy in the treatment of localized small cell carcinoma of the lung: A randomized prospective study by the Southeastern Cancer Study Group. Cancer 1981;47:2407-13.

17. Sas-Korczyńska B, Korzeniowski S, Wójcik E. Comparison of the effectiveness of "late" and "early" prophylactic cranial irradiation in patients with limited-stage small cell lung cancer. Strahlenther Onkol 2010;186;315-9.

18. Sas-Korczyńska B, Łuczyńska E, Chudyba A, et al. The retrospective evaluation of prophylactic cranial irradiation in patients treated for limited stage small-cell lung cancer a single centre study. Nowotwory J Oncol 2018;68:232-9.

19. Qiu G, DU X, Zhou X. Prophylactic cranial irradiation in 399 patients with limited-stage small cell lung cancer. Oncol Lett 2016;11:2654-60.

20. Faivre-Finn C, Snee M, Ashcroft L, et al. Concurrent once-daily versus twice-daily chemoradiotherapy in patients with limited-stage small-cell lung cancer (CONVERT): an open-label, phase 3, randomised, superiority trial. Lancet Oncol 2017;18:1116-25.

21. Slotman B, Faivre-Finn C, Kramer G, et al. Prophylactic cranial irradiation in extensive small-cell lung cancer. $\mathrm{N}$ Engl J Med 2007;357:664-72.

22. Takahashi T, Yamanaka T, Seto T, et al. Prophylactic cranial irradiation versus observation in patients with extensive-disease small-cell lung cancer: a multicentre, randomised, open-label, phase 3 trial. Lancet Oncol 2017;18:663-71.

23. Suzuki R, Komaki R. Is prophylactic cranial irradiation indicated for patients with extensive-stage small cell lung cancer with a complete response to first-line treatment? Radiother Oncol 2018;127:339-43.

24. Horn L, Mansfield AS, Szczęsna A, et al. First-Line Atezolizumab plus Chemotherapy in Extensive-Stage Small-Cell Lung Cancer. N Engl J Med 2018;379:2220-9.

25. Yang DX, Jairam V, Park HS, et al. Use of prophylactic cranial irradiation in patients with extensive-stage small cell lung cancer receiving immunotherapy. J Clin Oncol 2020;38:e19309.

26. Lee JS, Umsawasdi T, Lee YY, et al. Neurotoxicity in long-term survivors of small cell lung cancer. Int J Radiat Oncol Biol Phys 1986;12:313-21.

27. Bennett BM, Wells JR, Panter C, Yuan Y, Penrod JR. The humanistic burden of small cell lung cancer (SCLC): a systematic review of health related quality of life (HRQoL) literature. Front Pharmacol 2017;8:339.

28. Sun A, Bae K, Gore EM, et al. Phase III trial of prophylactic cranial irradiation compared with observation in patients with locally advanced non-small-cell lung cancer: neurocognitive and quality-of-life analysis. J Clin Oncol 2011;29:279-86.

29. Slotman BJ, Mauer ME, Bottomley A, et al. Prophylactic cranial irradiation in extensive disease small-cell lung cancer: short-term health-related quality of life and patient reported symptoms: results of an international Phase III randomized controlled trial by the EORTC Radiation Oncology and Lung Cancer Groups. J Clin Oncol 2009;27:78-84.

30. Le Péchoux C, Laplanche A, Faivre-Finn C, et al. Clinical neurological outcome and quality of life among patients with limited small-cell cancer treated with two different doses of prophylactic cranial irradiation in the intergroup phase III trial (PCI99-01, EORTC 22003-08004, RTOG 0212 and IFCT 99-01). Ann Oncol 2011;22:1154-63.

31. Arriagada R, Le Chevalier T, Borie F, et al. Prophylactic cranial irradiation for patients with small cell lung cancer in complete remission. J Natl Cancer Inst 1995;87:183-90.

32. Gregor A, Cull A, Stephens RJ, et al. Prophylactic cranial irradiation is indicated following complete response to induction therapy in small cell lung cancer: results of a multicentre randomised trial. United Kingdom Coordinating Committee for Cancer Research (UKCCCR) and the European Organization for Research and Treatment of Cancer (EORTC). Eur J Cancer 1997;33:1752-58.

33. Péchoux CL, Sun A, Slotman B et al. Prophylactic cranial irradiation for patients with lung cancer. Lancet Oncol 2016;17:e277-93.

34. Lok BH, Ma J, Foster A, et al. Factors influencing the utilization of prophylactic cranial irradiation in patients with limited-stage small cell lung cancer. Adv Radiat Oncol 2017;2:548-54.

35. Simó M, Vaquero L, Ripollés $P$, et al. Longitudinal brain changes associated with prophylactic cranial irradiation in lung cancer. J Thorac Oncol 2016;11:475-86.

36. Gondi V, Pugh SL, Tome WA, et al. Preservation of memory with conformal avoidance of the hippocampal neural stem-cell compartment during whole-brain 
radiotherapy for brain metastases (RTOG 0933): a Phase II multi-institutional trial. J Clin Oncol 2014;32:3810-6.

37. Redmond KJ, Hales RK, Anderson-Keightly H, et al. Prospective study of hippocampal-sparing prophylactic cranial irradiation in limited-stage small cell lung cancer. Int J Radiat Oncol Biol Phys 2017;98:603-11.

38. Guo WL, He ZY, Chen Y, et al. Clinical features of brain metastases in small cell lung cancer: an implication for hippocampal sparing whole brain radiation therapy. Transl Oncol 2017;10:54-8.

39. Kundapur V, Ellchuk T, Ahmed S, et al. Risk of hippocampal metastases in small cell lung cancer patients at presentation and after cranial irradiation: a safety profile study for hippocampal sparing during prophylactic or therapeutic cranial irradiation. Int J Radiat Oncol Biol Phys 2015;91:781-6.

40. Yeo SG. Perihippocampal metastasis following hippocampus-avoiding prophylactic cranial irradiation for small cell lung cancer: a case report. Onco Targets Ther 2017;10:4017-21.

41. Belderbos J, de Ruysscher D, DeJaeger K, et al. The

Cite this article as: Suwinski R. Prophylactic cranial irradiation in SCLC. Transl Lung Cancer Res 2021;10(4):20712078. doi: 10.21037/tlcr-2020-rtm-05 incidence and location of brain metastases following HAPCI compared with standard PCI in small cell lung cancer (SCLC) : A phase III trial. Int J Radiat Oncol Biol Phys 2019;105:S35.

42. Rodriguez De Dios N, Murcia M, Counago F, et al. Phase III trial of prophylactic cranial irradiation with or without hippocampal avoidance for small-cell lung cancer. Int J Radiat Oncol Biol Phys 2019;105;S35-6.

43. Manapov F, Käsmann L, Roengvoraphoj O, et al. Prophylactic cranial irradiation in small-cell lung cancer: update on patient selection, efficacy and outcomes. Lung Cancer (Auckl) 2018;9:49-55.

44. Brown PD, Pugh S, Laack NN, et al. Memantine for the prevention of cognitive dysfunction in patients receiving whole-brain radiotherapy: a randomized, double-blind, placebo-controlled trial. Neuro Oncol 2013;15:1429-37.

45. Brown PD, Gondi V, Pugh S, et al. Hippocampal Avoidance During Whole-Brain Radiotherapy Plus Memantine for Patients With Brain Metastases: Phase III Trial NRG Oncology CC001. J Clin Oncol 2020;38:1019-29. 